\title{
Information Du Patient Sur Le Diagnostic, Les Examens Paracliniques Et Le Traitement Au Chu-Sylvanusolympio (Togo)
}

Kakpovi K.

Service de Rhumatologie, CHR Kara Tomdè, Kara-Togo

Koffi-Tessio Ves

Mouzou $P$.

Oniankitan $O$.

Mijiyawa $M$.

Service de Rhumatologie, CHU Sylvanus Olympio, Lomé-Togo

Fianyo E.

Tagbor $K C$.

Service de Rhumatologie, Hôpital de Bè, Lomé-Togo

Houzou P.

Service de Rhumatologie, CHU Kara, Kara-Togo

$\underline{\text { Doi:10.19044/esj.2021.v17n7p189 }}$

Submitted: 27 October 2020

Accepted: 21 December 2020

Published: 28 February 2021
Copyright 2021 Author(s)

Under Creative Commons BY-NC-ND

4.0 OPEN ACCESS

Cite As:

Kakpovi K., Koffi-Tessio Ves, Mouzou P., Oniankitan O., Mijiyawa M., Fianyo E., Tagbor KC. \& Houzou P. (2021). Information Du Patient Sur Le Diagnostic, Les Examens Paracliniques Et Le Traitement Au Chu-Sylvanusolympio (Togo). European Scientific Journal, ESJ, 17(7), 189. https://doi.org/10.19044/esj.2021.v17n7p189

\section{Résumé}

Objectifs :déterminer le degré d'informations données aux patients hospitalisés en matière de diagnostic, des examens paracliniques et du traitement dans des services de médecine et de chirurgie du CHU SylvanusOlympio à Lomé (Togo). Méthodes :il s'agit d'une étude transversale portant sur 113 patients âgés de plus de 15 ans, hospitalisés dans les services de médecine et de chirurgie du CHU - SylvanusOlympio du 1er août au 31 octobre 2009. Résultats : cent treize patients (53 hommes, $47 \%$ et 60 femmes, $53 \%$ ) ont participé à l'enquête. Leur âge moyen était de 40 ans. Cinquante-sept $(50,4 \%)$ étaient hospitalisés en médecine et $56(49,6 \%)$ en chirurgie. La durée moyenne d'hospitalisation des patients était de 22 jours. 
Au sujet du diagnostic, $84 \%$ des patients avaient reçu une information et 54\% estimaient avoir été bien informés. Soixante-treize pourcent des patients n'avaient posé aucune question. Concernant les examens paracliniques, 48\% des patients avaient reçu des informations sur leur but et $46 \%$ sur leurs résultats. Quatre-vingt-dix-huit pourcent des patients n'étaient pas informés des risques potentiels des explorations et $80 \%$ n'avaient posé aucune question. Les ordonnances n'étaient pas expliquées dans $40 \%$ des cas. Quarante pourcent des patients en ignoraient le but, $90 \%$ ignoraient les risques potentiels et $76 \%$ n'avaient posé aucune question. Soixante-huit pourcent des patients connaissaient leur droit à l'information, et $78 \%$ désiraient tout savoir sur leur état de santé. Conclusion: les patients semblaient mieux informés en matière de diagnostic qu'en matière d'explorations paracliniques et de traitement. Il existait un contraste entre leur désir d'être informés et l'absence de questions sur leur état de santé.

Mots clés : Information, Patients, Diagnostic, Traitement, Togo

\title{
Patient Information on Diagnosis, Paraclinical Examinations and Treatment at Sylvanusolympio Teaching Hospital (Togo)
}

Kakpovi K.

Service de Rhumatologie, CHR Kara Tomdè, Kara-Togo

Koffi-Tessio Ves

Mouzou $P$.

Oniankitan $O$.

Mijiyawa M.

Service de Rhumatologie, CHU Sylvanus Olympio, Lomé-Togo

Fianyo E.

Tagbor KC.

Service de Rhumatologie, Hôpital de Bè, Lomé-Togo

Houzou P.

Service de Rhumatologie, CHU Kara, Kara-Togo

\begin{abstract}
Objectives: Determine the degree of information given to hospitalized patients in matters of diagnosis, the complementary exams and treatment in services of medicine and surgery of theSylvanusOlympioteachinghospitalin Lome (Togo). Methods: This is a cross-sectional study of 113 patients over the age of 15, hospitalized in the services of medicine and surgery of SylvanusOlympioteachinghospital from August 1 to October 31, 2009. Results: Hundred thirteen patients (53 men, $47 \%$ and 60 women, 53\%)
\end{abstract}


participated to the investigation. Their middle age was 40 years. Fifty seven $(50,4 \%)$ were hospitalized in medicine and $56(49,6 \%)$ in surgery. The middle length of hospitalization of the patients was 22 days. About the diagnosis, $84 \%$ of the patients had received information and 54\% estimated they had been informed well. Seventy-three percent of the patients had not asked any question. Concerning the exams, $48 \%$ of the patients had received some information on their aim and $46 \%$ on their results. Ninety-eight percent of the patients were not informed of the risks and $80 \%$ did not ask any question. About the treatment, the orders were not explained in $40 \%$ of the cases. Forty percent of the patients ignored the aim, 90\% ignored the risks and 76\% did not put any question. Sixty eight percent of the patients knew their right to information, and $78 \%$ wanted to know everything about their state of health. Conclusion: The patients are informed well about the diagnosis contrary to the complementary exams and to the treatment. Otherwise, they don't ask any questions whereas they want to be informed on their state of health.

Keywords: Information, Patient, Diagnosis, Treatment, Togo

\section{Introduction}

L'information est un élément central dans la relation entre le médecin et le patient. Il constitue un droit pour le patient, un devoir pour le soignant et une nécessité pour les deux. L'implication du patient dans le processus thérapeutique par une information adéquate et la possibilité de choisir son traitement semble favoriser son adhésion, son confort, l'amélioration de son état et de sa santé (Robert, 2005). Dans un passé encore récent la médecine paternaliste laissait aux médecins le loisir d'informer ou de laisser le patient dans l'ignorance aussi bien en ce qui concerne sa maladie qu'en ce qui concerne le traitement (Salome et Martin, 2007). Aujourd'hui les patients ne peuvent plus s'enfermer. Le devoir des médecins est d'informer les patients sur les avantages et les dangers des procédés de diagnostic et de traitement. Le fossé entre les professionnels de santé et les patients doit être comblé. Toutes les disciplines doivent satisfaire à cette obligation (Lazorthes, 2000). Le patient revendique de plus en plus le droit à des informations de qualité sur sa maladie et les traitements possibles (Robert, 2005). Ce travail a eu pour but de déterminer le degré d'information donnée aux patients en matière de diagnostic, des examens paracliniques et du traitement dans un centre hospitalier togolais.

\section{Patients et méthodes}

Il s'agit d'une étude transversale menée du $1^{\text {er }}$ août au 31 octobre 2009 chez des patients hospitalisés dans les services de médecine et de chirurgie du Centre Hospitalier et Universitaire SylvanusOlympio. Ont été inclus dans 
l'étude les patients âgés de plus de 15 ans et ayant obtenu l'exéat pendant la période de l'étude. Le mode de recrutement était aléatoire. La collecte des données a été faite sur une fiche d'enquête anonyme comportant les données démographiques (âge, genre, niveau d'instruction), les informations sur le diagnostic (vous a-t-on révélé la cause de votre mal ?, avez-vous posé des questions sur votre maladie ?, a-t-on répondu à vos questions ?, avez-vous été bien informé par le personnel soignant ?), les informations sur les examens paracliniques (but, risques, résultats, déroulement, a-t-on répondu à vos questions ?, avez-vous été bien informé ?) et les informations sur le traitement (but, risques, durée, avez-vous posé des questions ?, a-t-on répondu à vos questions ?, avez-vous été bien informé ?). Nous avons procédé à la lecture du questionnaire au patient et l'avons rempli au fur et à mesure. L'analyse des données a été réalisée avec le logiciel Epi info version 6.04dfi. Le test de Khi carré a été utilisé pour comparer les variables qualitatives avec un seuil de signification de 0,05 .

\section{Résultats}

Cent treize patients (53 hommes, 47\% ; 60 femmes, 53\%) ont participé à l'enquête. Leur âge moyen était de 40 ans avec des extrêmes de 16 et 90 ans. Cinquante-sept patients $(50,4 \%)$ étaient hospitalisés en médecine et 56 $(49,6 \%)$ en chirurgie. La durée moyenne d'hospitalisation des patients était de 22 jours avec des extrêmes de 1 et 240 jours. Selon le niveau d'instruction, on comptait 21 analphabètes $(18,6 \%)$ et 92 instruits $(31 \%$ du niveau primaire, $43,4 \%$ du niveau secondaire et $7,1 \%$ du niveau universitaire). Trente pourcent des femmes contre $5 \%$ des hommes étaient analphabètes. Au sujet du diagnostic, $84 \%$ des patients avaient reçu une information et seulement $27 \%$ des patients avaient posé des questions concernant leur maladie. Le taux de patients qui avaient posé des questions concernant leur maladie, était croissante selon le niveau d'instruction $(\mathrm{p}=0,01)$. Cinquante-quatre pourcent des patients estimaient avoir été bien informés. Les raisons évoquées par les 82 patients $(73 \%)$ qui n'avaient pas posé de question étaient principalement l'absence de question (39\%) et l'indisponibilité du personnel soignant (16\%). La variation des réponses aux questions « Avez-vous posé des questions concernant votre maladie ? Avez-vous été bien informé sur votre maladie par le personnel soignant? » selon le niveau d'instruction était statistiquement significative $(\mathrm{p}=0,04)$ (Tableau I). 
Tableau I. Satisfaction des patients sur le diagnostic selon le niveau d'instruction

\begin{tabular}{|l|l|l|l|l|l|}
\hline & $\begin{array}{l}\text { Analphabète } \\
\%\end{array}$ & $\begin{array}{l}\text { Primaire } \\
\%\end{array}$ & $\begin{array}{l}\text { Secondaire } \\
\%\end{array}$ & $\begin{array}{l}\text { Universitaire } \\
\%\end{array}$ & $\begin{array}{l}\text { P } \\
\text { valeur }\end{array}$ \\
\hline Diagnostic donné & 81,0 & 77,1 & 81,6 & 100,0 & NS* \\
\hline Questions posées & 14,3 & 17,1 & 34,7 & 62,5 & 0,01 \\
\hline Questions répondues & 100,0 & 100,0 & 100,0 & 100,0 & NS* \\
\hline $\begin{array}{l}\text { Bien informé du point de vue } \\
\text { du patient }\end{array}$ & 40,0 & 58,0 & 50,0 & 100,0 & 0,04 \\
\hline
\end{tabular}

$* \mathrm{NS}=$ non significatif $(\mathrm{p} \geq 0,05)$

Concernant les examens paracliniques, $48 \%$ des patients avaient reçu des informations sur leur but et $46 \%$ sur leurs résultats. Seulement $12 \%$ des patients étaient informés sur le déroulement de ces examens et $98 \%$ des patients n'étaient pas informés des risques potentiels des explorations. Les raisons évoquées par les 90 patients $(80 \%)$ qui n'avaient posé aucune question étaient principalement l'absence de question (40\%) et la préoccupation par la douleur et leur état de santé (29\%). La variation des réponses aux questions selon le niveau d'instruction n'était pas statistiquement significative (Tableau II).

Tableau II. Satisfaction des patients sur les examens paracliniques dc selon le niveau d'instruction

\begin{tabular}{|c|c|c|c|c|c|}
\hline & $\begin{array}{l}\text { Analphabète } \\
\%\end{array}$ & $\begin{array}{l}\text { Primaire } \\
\%\end{array}$ & $\begin{array}{l}\text { Secondaire } \\
\%\end{array}$ & $\begin{array}{l}\text { Universitaire } \\
\%\end{array}$ & $\begin{array}{c}\mathrm{P} \\
\text { valeur }\end{array}$ \\
\hline But & 42,9 & 48,6 & 46,8 & 71,4 & NS* \\
\hline Risques & 00,0 & 00,0 & 03,2 & 00,0 & NS* \\
\hline Résultats & 61,1 & 42,4 & 41,3 & 57,1 & NS* \\
\hline $\begin{array}{lll}\begin{array}{l}\text { Information } \\
\text { déroulement }\end{array} & \text { sur } & \text { le } \\
\end{array}$ & 05,8 & 16,6 & 09,7 & 16,6 & NS* \\
\hline Questions répondues & 100,0 & 100,0 & 100,0 & 100,0 & NS* \\
\hline $\begin{array}{l}\text { Bien informé du point de vue } \\
\text { du patient }\end{array}$ & 27,8 & 38,7 & 45,5 & 57,1 & NS* \\
\hline
\end{tabular}

$* \mathrm{NS}=$ non significatif $(\mathrm{p} \geq 0,05)$

Les ordonnances médicales avaient été expliquées à 64 patients (59,2\%) (33 patients avant l'achat des médicaments et 31 après l'achat des médicaments). Elles n'avaient pas été expliquées à 44 patients $(40,8 \%)$. Quarante pourcent des patients en ignoraient le but, 90\% ignoraient les risques potentiels. Cinquante-cinq pourcent des patients qui n'avaient pas honoré un examen paraclinique ou un traitement n'avaient pas été informés des conséquences encourues. Quarante des 109 patients $(36,7 \%)$ ayant répondus à la question sur la durée de leur traitement avaient été informés, contre 69 $(63,3 \%)$. Les raisons évoquées par les 86 patients $(76 \%)$ qui n'avaient posé aucune question étaient principalement l'absence de question (37\%), l'indisponibilité du personnel soignant (19\%) et la préoccupation par leur état de santé $(19 \%)$. La variation des réponses était croissant selon le niveau d'instruction chez les $24 \%$ qui avaient posé des questions et statistiquement significative ( $\mathrm{p}=0,004$; tableau III). 
Tableau III. Satisfaction des patients sur le traitement selon le niveau d'instruction

\begin{tabular}{|c|c|c|c|c|c|}
\hline & $\begin{array}{l}\text { Analphabète } \\
\%\end{array}$ & $\begin{array}{l}\text { Primaire } \\
\%\end{array}$ & $\begin{array}{l}\text { Secondaire } \\
\%\end{array}$ & $\begin{array}{l}\text { Universitaire } \\
\%\end{array}$ & $\begin{array}{l}\mathrm{P} \\
\text { valeur }\end{array}$ \\
\hline Ordonnances expliquées & 052,4 & 062,9 & 054,5 & 087,5 & NS* \\
\hline But & 057,1 & 062,9 & 055,6 & 087,5 & NS* \\
\hline Risques & 009,5 & 008,8 & 006,8 & 025,0 & NS* \\
\hline $\begin{array}{l}\text { Durée } \\
\text { Questions posées }\end{array}$ & $\begin{array}{l}033,3 \\
004,8\end{array}$ & $\begin{array}{l}033,3 \\
017,1\end{array}$ & $\begin{array}{l}038,3 \\
030,6\end{array}$ & $\begin{array}{l}050,0 \\
062,5\end{array}$ & $\begin{array}{l}\text { NS* } \\
0,004\end{array}$ \\
\hline Questions répondues & 100,0 & 100,0 & 100,0 & 100,0 & NS* \\
\hline $\begin{array}{l}\text { Refus d'un traitement ou } \\
\text { d'unexamen accordé }\end{array}$ & 050,0 & 100,0 & 100,0 & 100,0 & NS* \\
\hline $\begin{array}{l}\text { Conséquences d'un refus } \\
\text { expliquées }\end{array}$ & 050,0 & 025,0 & 075,0 & 000,0 & NS* \\
\hline $\begin{array}{l}\text { Bien informé du point de vue } \\
\text { du patient }\end{array}$ & 038,1 & 041,4 & 047,7 & 037,5 & NS* \\
\hline
\end{tabular}

* NS = non significative $(\mathrm{p} \geq 0,05)$

Quarante-quatre pourcent des patients estimaient avoir été bien informés. Soixante-huit pourcent des patients connaissaient leur droit à l'information contre $32 \%$ dont $13 \%$ pensaient que c'est une faveur (Tableau IV).

Tableau IV. Désir d'information du patient et sa connaissance du droit à l'information en fonction du niveau d'instruction

\begin{tabular}{|l|l|l|l|l|l|}
\hline & $\begin{array}{l}\text { Analphabète } \\
\%\end{array}$ & $\begin{array}{l}\text { Primaire } \\
\%\end{array}$ & $\begin{array}{l}\text { Secondaire } \\
\%\end{array}$ & $\begin{array}{l}\text { Universitaire } \\
\%\end{array}$ & $\begin{array}{c}\text { P } \\
\text { valeur }\end{array}$ \\
\hline $\begin{array}{l}\text { Connaissance du patient, de } \\
\text { son droit à l'information }\end{array}$ & 47,6 & 60,0 & 77,6 & 100,0 & 0,01 \\
\hline $\begin{array}{l}\text { Connaissance du patient, du } \\
\text { devoir d'information du } \\
\text { médecin }\end{array}$ & 23,8 & 54,3 & 73,5 & 100,0 & 0,0001 \\
\hline $\begin{array}{l}\text { Désir du patient de tout savoir } \\
\text { sur son état de santé }\end{array}$ & 68,4 & 79,4 & 81,3 & 75,0 & $\mathrm{NS*}$ \\
\hline
\end{tabular}

$* \mathrm{NS}=$ non significatif $(\mathrm{p} \geq 0,05)$

Soixante-huit patients (60\%) savaient que c'est une obligation pour le soignant d'informer son patient et $78 \%$ désiraient tout savoir sur leur état de santé même en cas de diagnostic grave ou de mauvais pronostic et ce quel que soit le sexe et le niveau d'instruction.

\section{Discussion}

Cette étude témoigne de l'insuffisance des informations données à nos patients concernant le diagnostic, les examens paracliniques et le traitement. $\mathrm{Au}$ sujet du diagnostic, $84 \%$ des patients avaient reçu une information et $54 \%$ estimaient avoir été bien informés. Soixante-treize pourcent des patients n'avaient posé aucune question. Concernant les examens paracliniques, $48 \%$ des patients avaient reçu des informations sur leur but et $46 \%$ sur leurs résultats. Quatre-vingt-dix-huit pourcent des patients n'étaient 
pas informés des risques potentiels et $80 \%$ n'avaient posé aucune question. Les ordonnances n'étaient pas expliquées dans $40 \%$ des cas. Quarante pourcent des patients en ignoraient le but, $90 \%$ ignoraient les risques potentiels et $76 \%$ n'avaient posé aucune question. Cinquante-cinq pourcent des patients qui n'avaient pas honoré un examen paraclinique ou un traitement n'avaient pas été informés des conséquences encourues. Soixante-huit pourcent des patients connaissaient leur droit à l'information, soixante pourcent savaient que le devoir d'informer pèse sur le soignant et $78 \%$ désiraient tout savoir sur leur état de santé. L'interprétation rigoureuse des résultats de cette étude impose la prise en compte de ses insuffisances liées aux biais de recrutement. Il s'est agi d'une enquête hospitalière qui n'a pris en compte que les patients hospitalisés dans certains services médicaux et chirurgicaux. Or, ces services ne sont pas les seuls à suivre ces étapes de diagnostic, d'examens paracliniques et de traitement; de plus ces étapes sont suivies chez tout patient y compris les patients vus en ambulatoire et dans chaque structure sanitaire du Togo. D'autre part, le fait d'interroger les patients à l'intérieur de la structure d'accueil et dans le service où ils ont été soignés pourrait biaiser les résultats obtenus. Le choix d'un tel échantillon ne pouvait donc permettre de généraliser les résultats obtenus à l'ensemble du pays. En dépit de ces insuffisances, notre étude garde tout son intérêt.

Notre échantillon comportait $53 \%$ de femmes et $47 \%$ d'hommes. Ceci reflète la structure de la population togolaise qui comporte $51 \%$ de femmes et $49 \%$ d'hommes (Direction Générale de la Statistique et de la Comptabilité Nationale, 2009). La composition de notre échantillon selon le niveau d'instruction révélait une prédominance du niveau d'instruction secondaire $(43,4 \%)$ et une faible proportion des patients du niveau universitaire. Ceci s'explique par un fort taux d'abandon des études au collège et au lycée. Ainsi, seulement $7 \%$ environ des patients interrogés avaient le niveau d'instruction universitaire. Mais cela pourrait s'expliquer aussi par le fait que les gens les plus instruits étant plus aisées(Rapport mondial, 2006), préfèrent peut-être se faire soigner dans les cliniques privées. Trente pourcent des femmes contre $5 \%$ des hommes sont analphabètes dans notre échantillon, ce qui reflète la disparité entre les genres en matière d'alphabétisation dans la sous-région (UNESCO, 2006).

Au sujet du diagnostic, nos résultats sont similaires à ceux trouvés en Espagne (Batista, 1994) en Allemagne (Dubachet von Rechenberg, 1977). Selon la même étude, la compréhension du diagnostic était influencée par la profession, l'âge et le genre. Notre étude montre par contre que la variation des réponses n'était pas influencée par le genre mais par le niveau d'instruction. Ceci pourrait s'expliquer par un complexe d'infériorité qu'éprouveraient les moins instruits vis-à-vis des soignants. L'absence de question $(39,2 \%)$ était la principale raison pour lesquelles les patients ne 
posaient de questions. Ceci pourrait signifier que l'information sur le diagnostic n'était pas du tout comprise. Dans le contexte africain, avant la colonisation, la médecine était exercée par les guérisseurs traditionnels qui jusqu'à ce jour constituent un élément de la culture africaine (Lazorthes, 2000). La maladie était considérée comme un mauvais sort pouvant venir soit des dieux (en guise de châtiment), soit des esprits des ancêtres, soit des sorciers malveillants. Le patient qui s'adressait à un sorcier guérisseur était avant tout informé sur l'origine de son mal. La colonisation a apporté avec elle la médecine occidentale. Avec la barrière linguistique comme toile de fond, l'information du patient était presque inexistante. Une autre cause de la médiocrité de l'information du patient est l'absence de la culture de l'évaluation. Or, cela manque à l'Afrique et beaucoup d'institutions publiques et privées n'ont pas encore cette culture d'autoévaluation. C'est le cas du CHU SylvanusOlympio qui ne dispose pas encore d'un système de contrôle de la qualité des soins. La vulgarisation des outils de l'information ces dernières années fait que le patient instruit devient de plus en plus demandeur d'informations. Parfois, avant de consulter, il a pu glaner sur Internet des informations sur sa pathologie, informations qu'il voudrait vérifier avec son médecin. Ceci pourrait expliquer pourquoi dans notre étude les patients les plus instruits sont ceux qui posent le plus de questions et qui sont les plus informés. Face à plus de $40 \%$ des adultes analphabètes en Afrique subsaharienne (Rapport mondial, 2006), le problème de l'information demeure presque entier.

Selon une étude faite en Grande Bretagne (Reynolds,1978), 24\% des interrogés n'étaient pas suffisamment informés sur le but des investigations (52\% dans notre étude) et $38 \%$ étaient mal informés au sujet des résultats de ces investigations (54\% dans notre étude). Dans une étude au Canada (Charles et al., 1994), 20\% des patients n'avaient pas été informés des résultats des investigations et $36 \%$ n'étaient pas informés sur les effets secondaires de ces examens notamment la douleur attendue (98,6\% dans notre étude). Nous notons des écarts assez importants entre nos résultats et les études précédentes. Les résultats de notre enquête montrent que les ordonnances médicales étaient expliquées dans 59,2\% des cas contre 86,7\% dans l'étude faite par Makoul (Makoul et al., 1995) à Chicago (USA). Les effets secondaires des médicaments n'avaient pas été signifiés à $23,6 \%$ des patients à Harvard (Cleary et al., 1991); à $20 \%$ au Canada (Charles et al., 1994) et à 49\% au Pakistan (Imam et al., 2007) contre 90\% dans notre étude. Il ressort de ces résultats que les patients du continent nord-américain semblaient être mieux informés sur les risques du traitement par rapport à ceux d'Asie et d'Afrique. Cependant, la taille de notre échantillon et l'absence d'études similaires dans la sous-région ne permettent pas de généraliser ces résultats. Dans le contexte africain, les informations données au patient par le guérisseur concernaient les 
doses et surtout les interdits. Les conséquences du non-respect de ces interdits n'étaient pas toujours expliquées. La notion d'effets indésirables était presque inconnue. Le patient était tenu de respecter scrupuleusement les consignes du guérisseur. Ces pratiques ont souvent été évoquées par certains auteurs africains (Hampaté Bâ, 1973 ; Camara Laye, 1953). Avant de pouvoir le faire, une étude comparative plus étendue est nécessaire. Il faut également tenir compte du fait qu'aux USA (Cleary et al., 1991) et au Canada (Charles et al., 1994), les prescripteurs étaient probablement des médecins alors que chez nous ce n'est pas toujours le cas: les assistants médicaux et même les infirmiers prescrivent parfois des ordonnances. D'autre part, il se pourrait que dans notre étude il n'y ait pas de risques potentiels du traitement dans beaucoup de cas et c'est pourquoi ces patients n'étaient pas informés. Soixante-seize pourcent des interrogés n'avaient pas posé de question dans notre étude tandis que Sleath (Sleath et al., 1999) en Caroline du Nord (USA) avaient trouvé $47 \%$. Dans notre étude, le mutisme était d'autant plus élevé que le niveau d'instruction était bas. Dans l'ensemble, $44,4 \%$ des patients estimaient avoir été bien informés sur leur traitement, contre 91,5\% dans l'étude de Brogiene et Gurevicius en Lituanie (Brogiene et Gurevicius, 2009). Cette différence entre les chiffres peut s'expliquer par trois choses : la barrière linguistique, le patient qui ne pose pas de question et le soignant qui pense que le patient n'a pas besoin de toutes ces informations. En effet, la non maitrise par le personnel médical de termes médicaux appropriés dans les langues locales, constitue un obstacle non négligeable au passage de l'information entre médecin et patient. Parfois, la dépendance économique vis-à-vis de la famille 'infantilise' le patient qui s'en remet à celle-ci pour les grandes décisions concernant sa santé. Il n'est donc pas surprenant que dans cette situation il pense que le soignant devrait adresser toutes les informations à la famille plutôt qu'à lui.

En Afrique, le patient ignore tout du diagnostic, des investigations et du traitement. Le médecin avait un pouvoir absolu sur le patient. Depuis les indépendances, tous les Etats africains ont placé la santé des populations parmi leurs priorités. Ainsi, la multiplication des centres de santé et la formation en grand nombre du personnel médical local ont contribué à améliorer les relations médecin - patient. Cependant, les principes d'information du patient n'ont pas beaucoup évolués. Ceci, pour des raisons essentiellement culturelles propres à l'Afrique. En effet, d'autre part, dans la culture africaine en général, il est inconvenant de poser des questions à celui qui vous rend service. Le manque de courtoisie de la part des soignants entretient cette culture et augmente le fossé qui sépare ces derniers de leurs patients. Ceci se reflète dans notre étude qui a montré que $73 \%$ des patients n'avaient pas posé de question concernant le diagnostic, $80 \%$ n'en ont pas 
posé sur les examens paracliniques et $76 \%$ ne l'ont pas fait concernant le traitement.

Le fait qu'une forte proportion de patients (60\% dans notre étude) sache qu'elle a droit à l'information et désire l'obtenir fait craindre la survenue de litige pour défaut d'information si rien n'est fait pour améliorer la qualité de l'information donnée aux patients. En Suisse (Langewitz et al., 2006), 96\% des patients désiraient être informés même en cas de mauvaises nouvelles contre $78 \%$ dans notre étude. La différence entre les chiffres pourrait s'expliquer par le fait qu'en Europe les gens désirent mettre de l'ordre dans leur vie avant de mourir et de ce fait, ils sont intéressés par toute information ayant trait à la fin de leur vie. En Afrique, l'absence de la culture de l'écriture et la superstition font que beaucoup pensent qu'en parlant de la mort on l'invite et ont peur de rédiger leur testament.

\section{Conclusion}

Les patients semblaient mieux informés en matière de diagnostic qu'en matière d'explorations paracliniques et de traitement. Il existait un contraste entre leur désir d'être informés et l'absence de questions sur leur état de santé.

\section{Déclaration de liens d'intérêt}

Il n'y a aucun conflit d'intérêt.

\section{References:}

1. Robert, CN. (2005). Le droit de savoir. Revue Médicale Suisse; I: 1298-303

2. Salome, V., Martin, W. (2007). Les droits du patient. Paris, France, Fleurus: 396p.

3. Lazorthes, G. (2000). L'homme, la société et la médecine ; Sciences humaines et sociales $6^{\text {ème }}$ Ed . Paris, France, Elsevier/Masson: 476p.

4. Direction Générale de la Statistique et de la Comptabilité Nationale (2009). Estimation de la population 2000 à 2009 (Tableau S2: Estimations de population). Consulté le 06/04/2020 sur http://www.stattogo.org/index.php?option=com_content\&view=c ategory $\&$ layout $=$ blog $\& i d=41 \&$ Itemid $=97$.

5. Rapport mondial de suivi sur l'EPT (2006).L'alphabétisation, un enjeu vital. Aperçu régional: Afrique subsaharienne. Consulté le 10/07/2020sur http://www.unesco.org/education/GMR2006/full/afric a_fr.pdf .

6. UNESCO. Rapport mondial de suivi sur l'EPT 2006 ; l'alphabétisation, un enjeu vital. Aperçu régional: Afrique 
subsaharienne. Consulté le 10/07/2020 sur http://www.unesco.org/education/GMR2006/full/africa_fr.pdf.

7. Batista, JE., Isabal, H., Macià, N., Palou, A. (1994). Survey of information provided to hospitalized patients at a monographic center. ActasUrolEsp; 18(7): 709-13.UNESCO.

8. Dubach, UC., Von Rechenberg, KN. (1977). Patient's understanding of their illness and patient-doctor relationship in out patient clinic. Dtsch Med Wochenschr ; 102(35): 1239-44.

9. Reynolds, M. (1978). No news, is bad news: patients' views about communication in hospital. Br Med J; 1(6128): 1673-6.

10. Charles, C., Gauld, M., Chambers, L., O'Brien, B., Haynes, RB., and Labelle, R. (1994). How was your hospital stay? Patients report about their care in Canadian hospitals. CMAJ; 150(11) : 1813-22.

11. Makoul, G., Arntson, P., Schofield, T. (1995). Health promotion in primary care: physician-patient communication and decision making about prescription medications. SocSciMed; 41(9) : 1241-54.

12. Cleary, PD., Edgman-Levitan, S., Roberts, M., Moloney, TW., McMullen, W., Walker, DJ., Delbanco, TL. (1991). Patients evaluate their hospital care: a national survey. Health Aff (Millwood); 10(4): 254-67.

13. Imam, SZ., Syed, KS., Ali, SA., Ali, SU., Fatima, K., Gill, M., Hassan, MO., Hashmi, SH., Siddiqi, MT., Khan, HM., Jameel, OF. (2007). Patients satisfaction and opinions of their experiences during admission in a tertiary care hospital in Pakistan - a cross sectional study. BMC HealthServRes ; 7 : p 161.

14. Hampaté Bâ, A. (1973). L'étrange destin de Wangrin ( $1^{\text {ère }}$ Ed). Paris, France, Edition 10/18: 384p.

15. Laye, C. (1953). L'enfant noir (1 $1^{\text {ère }}$ Ed). Paris, France, Plon : 224p.

16. Sleath, B., Roter, D., Chewing, B., Svarstad, B. (1999). Asking questions about medication : analysis of physician-patient interactions and physician perceptions. Med Care; 37 (11): 1169-73.

17. Brogiene, D., Gurevicius, R. (2009). Inpatients opinion on quality of Health care. Medicina (Kaunas); 45 (3) : 226-37.

18. Langewitz, W., Nübling, M., Weber, H. (2006). Hospital patients preferences for involvement in decision-making. A questionnaire survey of 1040 patients from a Swiss university hospital. Swiss Med Wkly ; 136(3-4) : 59-64. 